\title{
CONTROL OF CELL MEMBRANE FUSION BY LIPID COMPOSITION
}

\author{
David S. Roos \\ Department of Biological Sciences \\ Stanford University \\ Stanford, CA 94305
}

\section{INTRODUCTION AND OVERVIEW}

The fusion of biological membranes is an integral component of many physiological processes, occurring within and between cells in numerous situations of interest to such diverse fields as cell biology, developmental physiology, and medical pathology (reviewed in Poste and Nicholson, 1978; Lucy, 1982; Sowers, 1987). Observations of multinucleated cells in vertebrate tissues were first made by medical pathologists as early as the mid-19th century (reviewed by Langerhans, 1868), and cell fusion has continued to intrigue morphologists ever since, yet the precise molecular mechanisms governing the fusion of biological membranes remain unclear. In recent years, cell and membrane fusion techniques have been used extensively for the advancement of modern cell genetics (Davidson, 1977). Further development and refinement of applications for the biotechnology industry has lent increasing impetus to investigations into the biophysical and biochemical bases of cell fusion and other membrane interactions (de St. Groth and Scheidegger, 1980; Furusawa, 1980; Zimmermann, 1982; Gregoriades, 1984; Schlegel and Lieber, 1987).

The experiments reviewed below describe a genetic approach to the elucidation of membrane fusion mechanisms, based on examining the susceptibility or resistance of mutant cells to the polyene chemical fusogen polyethylene glycol $\left(\mathrm{PEG}^{*}\right)$. Biochemical and physiological investigation of a series of PEG fusion-resistant cell lines (Roos and Davidson, 1980) has demonstrated a correlation between cell membrane lipid composition (especially acyl-chain saturation) and the fusion response of a given cell culture (Roos and Choppin, 1985a). In further studies, careful manipulation of the lipid composition of these cells has allowed nearly complete control over the fusion phenotype (Roos and Choppin, 1985b), directly demonstrating the importance

*Abbreviations used in this report: Clone 1D, a 5-bromodeoxyuridine resistant mouse fibroblast cell line LMTK- Clone 1D (Kit et al., 1963); $\mathrm{F}_{8}, \mathrm{~F}_{16}, \mathrm{~F}_{40}$, fusion-resistant cell lines isolated from Clone 1D by 8, 16, or 40 cycles of polyethylene glycol-induced fusion (Roos and Davidson, 1980); MHV, murine hepatitis virus, strain A59; PEG, polyethylene glycol; S/P ratio, ratio of saturated to polyunsaturated fatty acyl chains; VSV, vesicular stomatitis virus, Indiana serotype. 
of membrane lipids in regulating cell fusion, and providing a useful approach to the control of cell fusion in various technological applications (Roos et al., 1987).

Initially, this project focused on cell fusion induced by polyethylene glycol, because of its convenience of use, reproducibility, absence of extraneous biological material, and importance to the development of fusion technology. Nevertheless, the results obtained with PEG apply equally well to other forms of membrane fusion, including virus-induced fusion and spontaneously occurring fusion. These findings suggest similarities in certain structural aspects, if not the precise mechanisms, of a variety of forms of membrane fusion. While the original studies were carried out in a particular system of cultured mouse fibroblasts, our experimental observations have now been found to apply to other cell systems as well (Roos et al., 1987). Further studies, briefly outlined but beyond the scope of this report, have taken advantage of the availability of the cell lines described here to demonstrate the importance of lipid composition to such diverse membrane-associated phenomena as immune recognition of tumor cells, metastatic spread of neoplastic disease, and the determination of virus host range.

\section{ISOLATION AND CHARACTERIZATION OF FUSION-ALTERED CELL LINES}

\section{PEG fusion-resistant mutants}

Individual cultured cell lines generally exhibit a characteristic response to a given fusogen such as PEG, but comparisons between cell lines have revealed considerable differences in the potential range of fusion response (Davidson and Gerald, 1976; Blow et al., 1978; Wang et al., 1982). Consideration of these observations suggested both the importance of working with a single, well-defined, clonal cell line, and the feasibility of isolating mutants which differ in their response to fusogenic treatment. The murine fibroblast cell line designated Clone 1D (Kit et al., 1963) has been extensively characterized with respect to its PEG fusion response (Davidson et al., 1976; Robinson et al., 1979) and provided a suitable parental strain for the studies described below. Although several different techniques for isolating PEG fusion-resistant mutants were attempted, the most successful (if laborious) approach involved repeated cycles of treatment with fusogenic concentrations of PEG ( $50 \%$ by weight) followed by outgrowth of the remaining unfused cells (Roos and Davidson, 1980). This repetitive protocol produced a series of cell lines designated $\mathrm{F}_{1}, \mathrm{~F}_{2}, \mathrm{~F}_{3}$, etc., where the subscripted number indicates the number of cycles of selection required to produce each cell line.

The susceptibility of these cell lines to PEG-induced fusion is illustrated in Figure 1. Typically, $>90 \%$ of the cells in PEG-treated Clone 1D cultures fuse into large, multinucleated syncytia. However, increasingly selected cell lines are progressively less susceptible to fusion: only $\sim 70 \%$ of the nuclei in $\mathrm{F}_{8}$ cultures are ultimately found in fused cells, $\mathrm{F}_{16}$ cells exhibit $\sim 50 \%$ fusion, and the $\mathrm{F}_{40}$ mutants are characterized by $<20 \%$ fusion. Thus, we have succeeded in isolating highly PEG fusion-resistant mutants from the original, fusible Clone 1D cell line. It has not, however, proved possible to generate mutants which are wholly resistant to the fusion-inducing effects of PEG (despite considerable effort in this direction), a finding which was suggested to result from insurmountable constraints governing viable cell architecture (Roos et al., 1983). The nature of these constraints can now be understood at the biochemical level, as will be discussed below.

Each of the PEG fusion-resistant cell lines is stable, maintaining its characteristic fusion response even when carried in culture for several months without PEG treatment or other selection (Roos et al., 1983). Each 


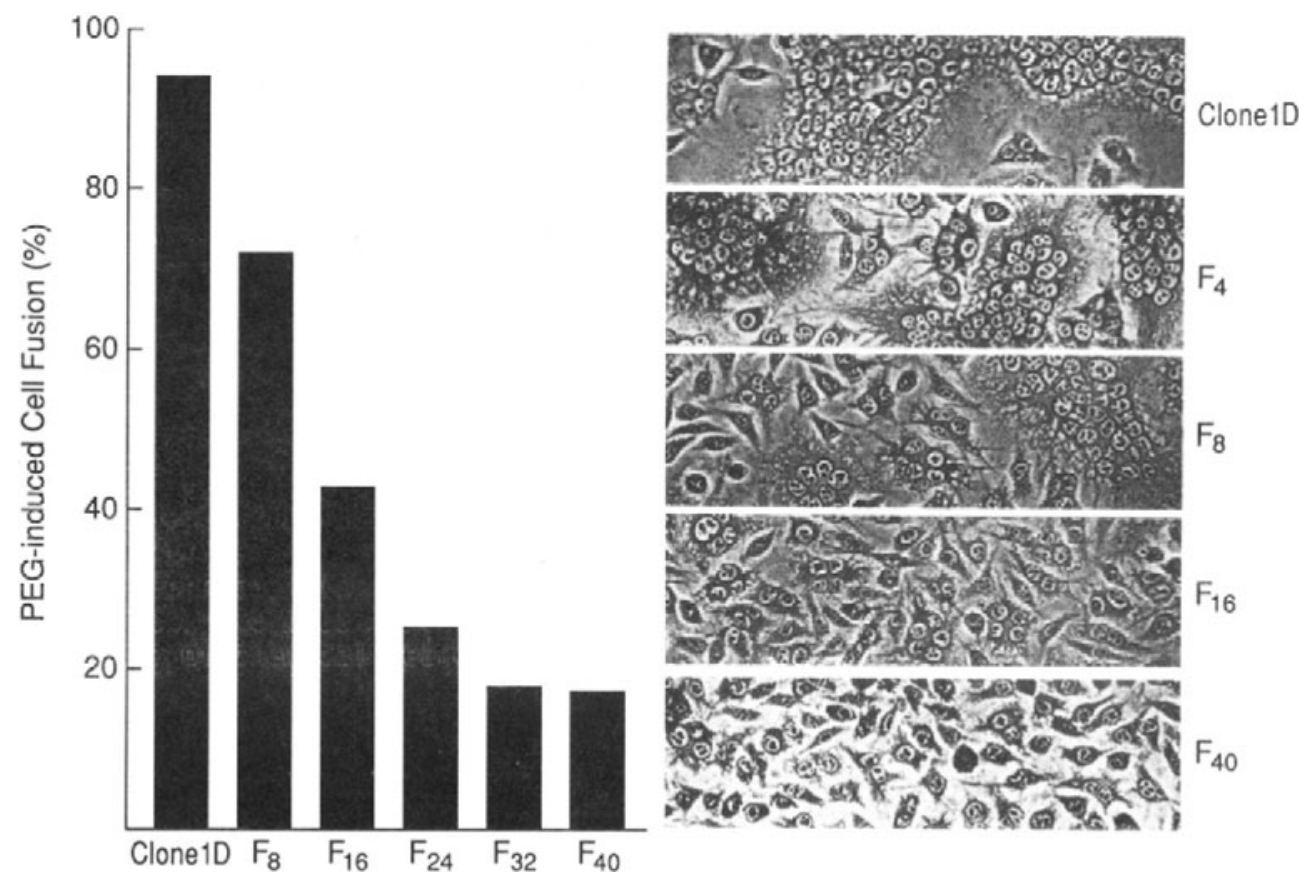

Figure 1. Response of Clone 1D and fusion-altered cell lines to PEG treatment. Confluent cultures of the parental cell line Clone 1D and PEG-resistant mutants were treated for 1 min with 50\% PEG-1000 as previously described (Roos and Davidson, 1980) and fixed and stained for microscopy. Fusion response was scored as the percentage of nuclei found in fused cells of any size. PEG fusion-resistant cell lines $\mathrm{F}_{4}, \mathrm{~F}_{8}, \ldots, \mathrm{F}_{40}$ were selected by repeated cycles of PEG treatment followed by outgrowth of the remaining unfused cells; subscripts indicate the number of cycles of selections required to produce each cell line. The selection protocol employed produced a series of stable, homogeneous cell lines each of which is slightly more resistant to PEG-induced fusion than its predecessor, as discussed in the text. Reproduced, with permission, from Roos et al. (1983) and Roos and Choppin (1985a).

cell line also appears to be homogeneous in its fusion response; examination of clonal cultures isolated from several different cell lines of intermediate susceptibility to PEG demonstrates that each of these lines consists of a population of cells which are uniformly reduced in fusibility (Roos and Davidson, 1980). The $\mathrm{F}_{8}$ cell line, for example, is composed of individual cells each of which is approximately one-half as likely to fuse as are the parental Clone 1D cells*, a finding which contrasts sharply with expectations for an intermediate stage in the selection of classical genetic mutants, where we might predict a mixed population containing fusible parental cells enriched with unfusible mutants. This observation has interesting implications for the genetic basis of fusion resistance.

\footnotetext{
${ }^{*}$ Combining the overall fusion response for a given cell culture (e.g. data from Figure 1) with observations of cell density, the degree of cell spreading, etc., it is possible to model the fusion response mathematically (Röhme and Thorburn, 1981; D.S. Roos and B. Singer, unpublished analyses). Applying such models yields an underlying probability of fusion for a single pair of adjacent Clone $1 \mathrm{D}$ cells $\approx 45 \%$, following a standard PEG treatment protocol. (The overall fusion response for Clone 1D is much higher [cf. Figure 1], because each cell contacts several adjacent cells, fusion with any one of which is sufficient to be scored as a fused cell.) The calculated probability that a closely apposed pair of $\mathrm{F}_{8}$ cells will fuse is $27 \%$, and the underlying fusion probabilities for $F_{16}$ and $F_{40}$ cells are $12 \%$ and $4 \%$, respectively.
} 


\section{PEG-fusion mutants are unusually susceptible to virus-induced fusion}

Having isolated the PEG fusion-resistant cell lines described above, it was of interest to characterize their response to other fusogenic treatments as well. Most enveloped viruses are capable of causing the fusion of biological membranes, as membrane fusion is a necessary step in their infectious pathway (Choppin and Scheid, 1980; White et al., 1983; Spear, 1987). Under appropriate conditions, many of these viruses are also capable of inducing fusion between cells. Several of the PEG-resistant cells lines have been examined for their susceptibility to fusion following treatment with various enveloped viruses, as shown in Table I.

Somewhat surprisingly, increasingly PEG-resistant cells are increasingly susceptible to fusion induced by a variety of enveloped viruses which cause fusion under different conditions (D.S. Roos, C. Stevenson, K.V. Holmes, and P.W. Choppin, manuscript submitted for publication). Vesicular stomatitis virus (VSV) readily infects these cells and causes fusion both by bridging adjacent cells with virus particles which subsequently fuse with each cell, and through the action of viral fusion proteins inserted in the surface membranes of infected cells. Although VSV-infected Clone 1D and $F_{40}$ cells produce similar titers of virus (not shown), Clone 1D cells are markedly less susceptible to VSV-induced fusion. Murine hepatitis virus (MHV) infects both Clone 1D and $\mathrm{F}_{40}$ cells and is capable of fusing both cell types, but the overall extent of fusion is much higher in $F_{40}$ cultures, at least in part because Clone 1D cells are unable to produce infectious virions. Sendai virus is unable to infect either cell line, but can cause fusion upon the addition of high titers of inactivated virus (sometimes called fusion from without; Bratt and Gallaher, 1969). Under these conditions as well, more extensive fusion was seen in $\mathrm{F}_{40}$ cells than in the Clone 1D cell line. Similar observations of increased susceptibility to virus-induced fusion in PEG-resistant cell lines have been made for many other viruses, including influenza, SV5, and visna virus (an ovine retrovirus).

Although the inverse correlation between chemically-induced and virusinduced fusion was unexpected, it is notable that the PEG-resistant cell lines, selected without any intentional reference to other charteristics, are nevertheless altered with respect to another membrane-associated phenomenon, namely their susceptibility to virus-induced fusion. Across the graded series of increasingly PEG fusion-resistant mutants, the progressive nature of fusion alteration holds up for all viruses tested (cf. $F_{16}$ data in Table I). The

Table I. Fusion Response of PEG-Resistant Cell Lines

\begin{tabular}{lccccc}
\hline & \multicolumn{5}{c}{ Percent fusion following indicated treatment } \\
\cline { 2 - 5 } Cell Line & PEG & VSV $^{*}$ & Sendai $^{\dagger}$ & MHV $^{\ddagger}$ & (spontaneous fusion) \\
\hline Clone 1D & 96 & $8(+)^{8}$ & $6(-)$ & $22(-)$ & 2 \\
$\mathrm{~F}_{16}$ & 58 & $\mathrm{ND}$ & $17(-)$ & $45(+)$ & 4 \\
$\mathrm{~F}_{40}$ & 24 & $20(+)$ & $20(-)$ & $64(+)$ & 5 \\
\hline * Indiana serotype; cell fusion following brief exposure to acid pH (White et al., 1981). \\
† Z-strain; fused 'from without' using inactivated virus (Holmes and Choppin, 1966). \\
₹ Strain A59; fusion carried out according to Holmes et al. (1981). \\
\& +/-indicates the ability/inability to produce infectious virions.
\end{tabular}


inverse relationship between PEG and virus response may explain the observation that BHK-21 cells, which have frequently been used for studies on virus-induced fusion because of their extreme sensitivity to many fusogenic viruses (Holmes and Choppin, 1966; Compans et al., 1966, Helenius et al., 1980; White et al., 1981), are among the most highly PEG fusion-resistant cell lines we have encountered (Wang et al., 1982).

Spontaneous fusion has also been examined in these cell lines (Roos and Choppin, 1985b), and also changes progressively across the series of increasingly fusion-altered mutants, as shown in the rightmost column of Table I. As found for virus-induced fusion, spontaneous fusion is inversely correlated with PEG fusion response in these cell lines. Although the overall level of spontaneous fusion is low in both Clone $1 \mathrm{D}$ and $\mathrm{F}_{40}$ cultures, differences between these cell lines have been reproducibly observed over several years of study and are statistically highly significant $(P<0.001)$.

\section{BIOCHEMICAL ANALYSIS OF FUSION-ALTERED CELL LINES}

The availability of closely related cell lines which differ so dramatically in their response to diverse fusogenic treatments provided an opportunity to explore the biochemical basis of the fusion alterations observed in these cells. As an added advantage, the existence of a graded series of stable mutants intermediate between Clone $1 \mathrm{D}$ and $\mathrm{F}_{40}$ has permitted the search for biochemical alterations to be narrowed, focusing on differences which are progressive across the entire series of cell lines isolated.

\section{Protein analysis of fusion-altered cells}

Initial efforts towards elucidating biochemical differences associated with the altered fusion response in these cells concentrated on the analysis of their protein composition (Roos, 1985). A small number of differences have been detected in two-dimensional electrophoretic separations of proteins isolated from Clone and $F_{40}$ cells, including a series of high molecular weight membrane glycoproteins which are increasingly abundant in increasingly fusion-altered cells. Differences have also been seen in protein glycosylation and growth inhibition by certain lectins. Overall, however, protein differences between Clone $1 \mathrm{D}$ and $\mathrm{F}_{40}$ are slight, and no functional properties are known for those differences which have been found. While antibodies can be prepared with slight specificity for either Clone 1D or $\mathrm{F}_{40}$ cells, these antibodies have no clear effect on the fusion response. Any direct association of membrane proteins with fusion is further called into question by experiments on the fusion of protease-treated cells: Clone $1 \mathrm{D}$ cells remained more highly susceptible to PEG-induced fusion than the $\mathrm{F}_{40}$ mutants even after exposure to a crude mixture of pancreatic proteases, a treatment which degraded the high molecular weight proteins described above, eliminated cellspecific antibody staining, and greatly diminished lectin binding*

While specific proteins must surely be involved at some level in generating the contrasting fusion responses of Clone $1 \mathrm{D}$ and $\mathrm{F}_{40}$ cells, differences in the lipid composition of these cell lines appear to be centrally involved in mediating the fusion alterations described above. An extensive analysis of the lipid composition of Clone $1 \mathrm{D}$ and $\mathrm{F}_{40}$ cells has previously been published (Roos and Choppin, 1985a), and will be summarized here only briefly.

\footnotetext{
*Fusion of protease-treated cells was performed in suspension or according to the 'pancake fusion' technique (O'Malley and Davidson, 1977).
} 


\section{Lipid composition is directly correlated with the fusion-altered phenotype}

Several aspects of the lipid composition of these cell lines fulfill the requirement of progressive change across the series of increasingly PEG fusion-resistant mutants, as summarized in Table II. Although these data are derived from biochemical analyses of whole cells, qualitatively similar results are obtained from membrane preparations (Roos, 1985).

The parental cell line, Clone $1 \mathrm{D}$, which is highly susceptible to PEGinduced fusion, contains approximately equal amounts of saturated and polyunsaturated fatty acyl chains $(\mathrm{S} / \mathrm{P}$ ratio $\approx 1.0)$, but the extent of fatty acid saturation increases progressively in increasingly PEG-resistant cells (S/P ratio $\approx 4.0$ in $\mathrm{F}_{40}$ cells). This change in acyl chain composition results from a general increase in the prevalence of all saturated species (palmitate, stearate, etc.) and a decline in the prevalence of most polyunsaturates (linoleic acid, arachidonate, etc.) (Roos and Choppin, 1985a). Fusion-altered cells also contain high levels of an unusual ether-linked neutral lipid (1-alkyl, 2,3diacyl glycerol; Snyder, 1972; Mangold and Paltauf, 1983). Neutral ether lipids (excluding plasmalogens) are virtually absent, from Clone 1D cells, but increase in prominence to rank second only to cholesterol as the major neutral lipid in $\mathrm{F}_{40}$ cells. The decreased ganglioside content in fusion-altered cells is intriguing, particularly because of its presence in membranes, but variations in ganglioside composition dependent on cell density and culture conditions overwhelm the differences between cell lines (Roos et al., 1987).

Acyl chain saturation and ether lipid content are both closely correlated with susceptibility to fusion across the series of mutant cell lines under study. In fact, these phenomena may be metabolically linked (Roos and Choppin, 1985a): ether-lipids are unusual among neutral lipids in being preferentially localized in cell membranes (Pfleger et al., 1968; Wood et al., 1970; Schroeder and Vagelos, 1976; Friedberg and Halpert, 1978), carrying with them the highly saturated acyl chains characteristic of neutral lipids (Spector et al., 1981).

\section{FunCtional Control of CELL FUSION BY LIPID CoMPOSITION}

Although the biochemical analyses of these cells reveal strong correlations between lipid composition and fusion response, such correlations are

Table II. Lipid Composition of Fusion-altered Cell Lines*

\begin{tabular}{lcccc}
\hline & \multicolumn{4}{c}{ Cell Line } \\
\cline { 2 - 5 } & Clone 1D & $\mathrm{F}_{8}$ & $\mathrm{~F}_{16}$ & $\mathrm{~F}_{40}$ \\
\hline PEG-induced fusion & 95 & 68 & 46 & 19 \\
Acyl chain saturation (S/P ratio) & 1.0 & 2.8 & 3.3 & 4.0 \\
Neutral ether-linked lipids (relative content) & 1 & 12 & 23 & 36 \\
Triglycerides (relative content) & 1 & 4 & 6 & 8 \\
Total gangliosides (relative content) & 4 & 3 & 3 & 1 \\
\hline
\end{tabular}

* For details of lipid analysis see Roos and Choppin (1985a). 
fundamentally circumstantial. To evaluate the functional relevance of these correlations, the lipid content of Clone 1D cells and its fusion-altered derivatives has been modified in several ways. Through the use of such 'lipidalteration' experiments it has been possible to directly demonstrate the importance of lipid composition in controlling cell membrane fusion.

\section{Alteration of acyl-chain saturation completely controls PEG-induced fusion}

The degree of lipid saturation in all of these cell lines is remarkably constant under normal culture conditions, but saturation can be experimentally controlled by growth in medium containing high concentrations of various fatty acids (presented as sodium salts, conjugated to serum albumin, or by more elaborate procedures), as shown in the upper panel of Table III. When Clone 1D cells were grown for 18 hours in the presence of $10^{-4}$ molar saturated fatty acid, the S/P ratio increased to $>3: 1$, similar to the highly saturated levels found in untreated $\mathrm{F}_{40}$ cultures. Growth in polyunsaturated fatty acids suppressed the acyl chain saturation of $F_{40}$ cells to levels to levels at or below those normally found in the parental cell line Clone 1D.

The effects of these changes on the fusion response of Clone $1 \mathrm{D}$ and $\mathrm{F}_{40}$ cells is illustrated in Figure 2. As already seen, control cultures of Clone 1D cells are highly susceptible to PEG-induced fusion (panel $2 A$ ), while little fusion occurs in similarly treated $\mathrm{F}_{40}$ cells (panel $2 B$ ). However, when the fatty acid saturation of Clone 1D cultures was altered to resemble $F_{40}$ by growth in high concentrations of saturated fatty acids (in this case nonadecanoic acid), the fusion response was abolished (panel $2 C$ ). Conversely, susceptibility to PEG-induced fusion was restored in $\mathrm{F}_{40}$ cells grown in polyunsaturated fatty acid (such as linolenic acid; panel $2 D$ ). Quantitatively, these treatments were capable of completely controlling PEG fusion, as shown in the second panel of Table III. A time course and dose response curves for fatty acid supplementation and PEG-induced fusion have previously been published (Roos and Choppin, 1985b).

Additional experiments have probed the biological limits of the PEG fusion response in these cells. Untreated Clone 1D cells are relatively high in polyunsaturated acyl chain content. However, when grown in the presence of linolenic acid (or other polyunsaturates), the S/P ratio of these cells was suppressed even below control levels (Table III, top panel). Accordingly, the cells in these cultures became even more highly sensitive to PEG-induced fusion than usual. As untreated Clone 1D cells normally fuse virtually to completion following a standard treatment with 50\% PEG (cf. Figure 1), the enhanced fusion of these cells was detected as a decline in the concentration of PEG required to stimulate fusion (Roos and Choppin, 1985b). By contrast, $\mathrm{F}_{40}$ cells could not be rendered more highly saturated than their usual state. Growth in high concentrations of saturated fatty acid was highly toxic to the $\mathrm{F}_{40}$ mutants, suggesting that these cells have reached the physiological limit of PEG fusion resistance: higher acyl chain saturation, which might be expected to produce a completely PEG-resistant line, simply kills the cells.

\section{Control of virus-induced fusion and spontaneous fusion by fatty acid supplements}

Virus induced fusion of Clone 1D cells and its PEG-resistant derivatives is inversely correlated with chemically mediated fusion, as already seen (Table I). Nevertheless, the same lipid supplement experiments which 
allowed control over the PEG fusion response also altered the susceptibility of these cells to virus-induced fusion (lower half of Figure 2; third panel of Table III). Murine hepatitis virus, the most fusogenic virus yet tested in this system, fuses $\mathrm{F}_{40}$ cells extensively while producing relatively little fusion of Clone $1 D$ cells (panels $2 F$ and $2 E$, respectively). Appropriate lipid supplements, however, were able to render Clone 1D cells susceptible to MHV fusion and $\mathrm{F}_{40}$ cells MHV-resistant (panels $2 G$ and $2 H$ ). Thus modification of cellular lipid chain saturation by growth in fatty acid supplemented medium is able to control the response of these cell lines to both chemical and viral fusogens in parallel, despite the fact that these two classes of fusogens exert opposite effects on the cells. Spontaneously occurring fusion, another phenomenon inversely related to the PEG fusion response, can also be controlled by fatty acid supplements, as seen in the bottom panel of Table III.

Table III. Control of Cell Fusion by Modification of Fatty Acid Composition

\begin{tabular}{|lccc|}
\hline & & \multicolumn{3}{c|}{ Lipid Supplement* } \\
\cline { 2 - 4 } Cell Line & polyunsaturated FA & control & saturated FA \\
\hline Clone 1D & 0.31 & 0.93 & $\longrightarrow$ \\
$\mathrm{F}_{40}$ & 0.54 & 2.93 & 2.80 \\
\hline
\end{tabular}

\begin{tabular}{|c|c|c|c|}
\hline \multirow[b]{2}{*}{ Cell Line } & \multicolumn{3}{|c|}{ Lipid Supplement* } \\
\hline & polyunsaturated FA & control & saturated FA \\
\hline Clone 1D & 91 & 93 & $\rightarrow \quad 17$ \\
\hline $\mathrm{F}_{40}$ & 89 & 20 & {$[13]^{\ddagger}$} \\
\hline
\end{tabular}

\begin{tabular}{|lrccc|} 
& \multicolumn{4}{c|}{ Lipid Supplement* } \\
\cline { 2 - 5 } Cell Line & polyunsaturated FA & control & saturated FA \\
\hline Clone 1D & 15 & 24 & 61 \\
$\mathrm{~F}_{40}$ & 9 & 56 & {$\left[^{\dagger}\right.$} \\
\hline
\end{tabular}

\begin{tabular}{|lrccc|}
\hline \multirow{2}{*}{ Cell Line } & $<2$ & \multicolumn{4}{c|}{ Lipid Supplement* } \\
\cline { 2 - 5 } & polyunsaturated FA & control & saturated FA \\
\hline Clone 1D & $<2$ & 2 & 15 \\
$\mathrm{~F}_{40}$ & $<$ & 5 & {$[20]^{\ddagger}$} \\
\hline
\end{tabular}

* Fatty acid supplements were $10^{-4}$ molar linolenic acid (18:3) or nonadecanoic acid (19:0), supplied as conjugates to delipidated serum albumin ( 1:1 ratio of FA to BSA).

$\dagger$ Spontaneous fusion subtracted to yield PEG-specific or virus-specific fusion.

$\ddagger$ Quantitation unreliable due to high toxicity in $\mathrm{F}_{40}$ cells treated with saturated fatty acid. 


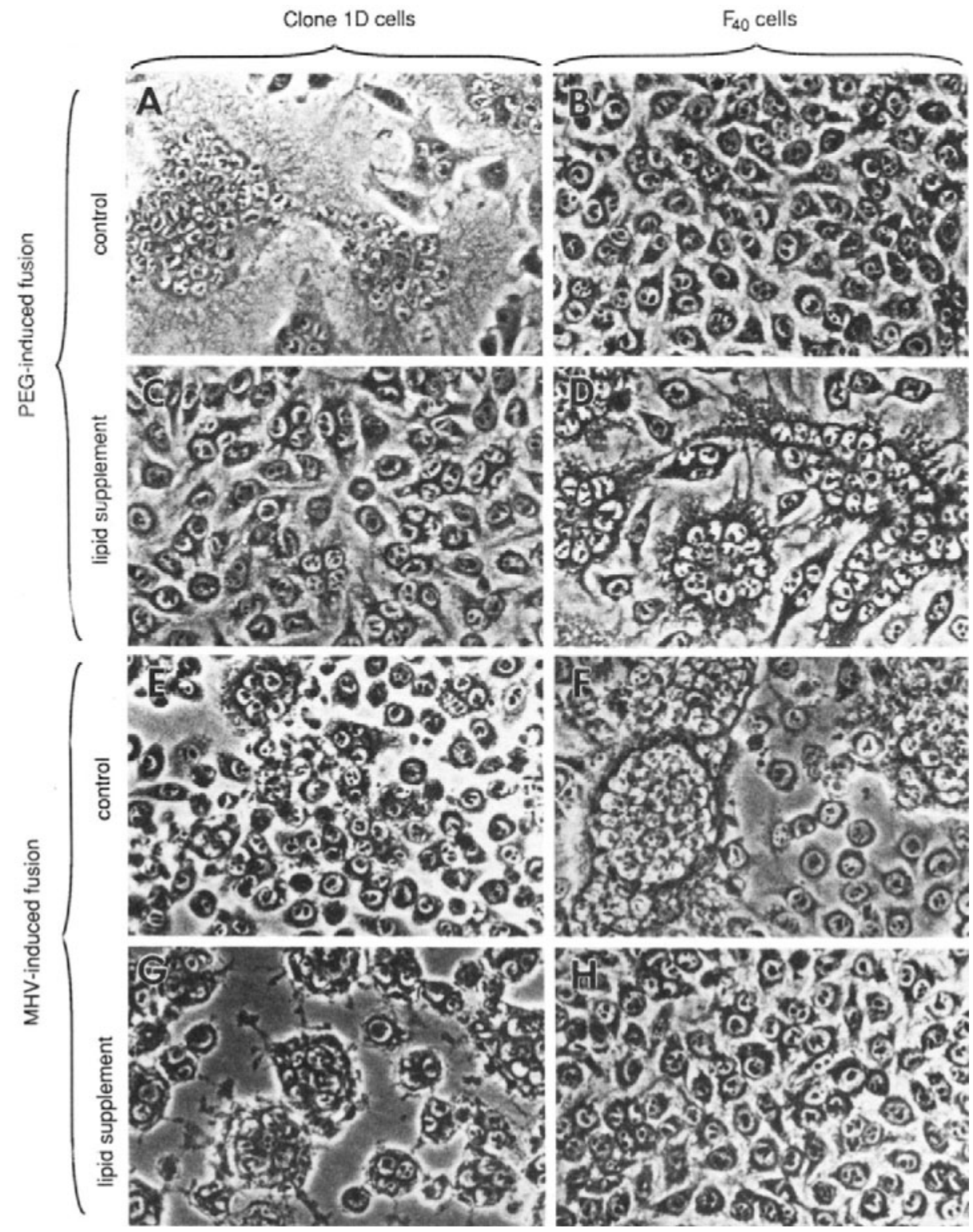

Figure 2. Control of cell fusion by lipid alteration. Confluent cultures of Clone 1D cells (panels $A, C, E$, and $G$ ) or $\mathrm{F}_{40}$ cells (zanels $B, D, F$, and $H$ ) were grown for $18 \mathrm{hr}$ in either control medium (panels $A, B, E$, and $F$ ) or medium supplemented with exogenous fatty acids conjugated to serum albumin (panels $C, D, G$, and $H$ ). Supplements were $10^{-4}$ molar nonadecanoic acid (19:0) for Clone 1D, and $10^{-4}$ molar linolenic acid (18:3) for $F_{40}$. Cultures were then treated with polyethylene glycol (PEG; panels A-D) or infected with murine hepatitis virus (MHV; panels $E-H$ ). See Roos and Choppin (1985b) for further experimental details.

Control cultures of Clone 1D are highly sensitive to PEG-induced fusion (panel $A$ ) but resistant to virus-induced fusion (panel $E$ ). $\quad F_{40}$ cells exhibit the reverse phenotype: they are PEG-resistant but sensitive to virus-induced fusion (panels $B$ and $F$, respectively). Growth of $F_{40}$ cells in high concentrations of polyunsaturated fatty acid, however, corrects the altered fusion response in these mutants, rendering them sensitive to PEG and virus-resistant (panels $D$ and $H$ ). Conversely, increasing the acyl chain saturation of Clone 1D cells mimics the PEG-resistant/virus-fusible phenotype characteristic of $\mathrm{F}_{40}$ (panels $C$ and $G$ ). In sum, both PEG-induced and virus-induced fusion can be completely controlled in this system, through the appropriate addition of lipid supplements to the growth medium. 


\section{Lipid composition and fusion response of tumor explants}

In addition to the fatty acid alterations found in $\mathrm{F}_{40}$ cells, neutral etherlipid content is elevated in these mutants, as noted above. While supplementing the growth medium with various fatty acids provides an effective method for controlling both acyl chain saturation and cell fusion, these experiments also result in parallel changes in ether-lipid concentration (Roos, 1985). Careful fluctuation analysis of a variety of lipid supplement experiments points to chain saturation, rather than ether-lipid content, as the principle factor in regulating fusion response, but more direct evidence for this conclusion comes from an unexpected source.

Following the observation of others that ether-linked lipids are frequently associated with a wide variety of animal tumors (Bollinger, 1967; Wood and Snyder, 1967; Snyder, 1972; Lin et al., 1978), we have used the series of cell lines described here to study the effects of lipid composition on tumor formation in mice (Roos and Choppin, 1984). For reasons known, perhaps, only to Mnemusyne*, growth of these cell lines as tumors produces tissue containing high levels of ether-lipid (similar to $\mathrm{F}_{40}$ cells grown in culture), while retaining a low ratio of saturated to polyunsaturated fatty acyl chains (similar to Clone 1D). Thus the prevailing conditions of in vivo growth in mice have fortuitously separated the phenomena of fatty acid saturation and etherlipid content more effectively than yet achieved by any experimental program in vitro. Table IV presents the results of biochemical analyses and tests for fusion response of cultured explants derived from these tumors.

Tumors arising from either Clone $1 \mathrm{D}$ or $\mathrm{F}_{40}$ cells exhibit the low $\mathrm{S} / \mathrm{P}$ ratio characteristic of Clone $1 \mathrm{D}$ cells, coupled with the high ether-lipid content characteristic of $\mathrm{F}_{40}$. Although it is not possible to directly assess susceptibility to PEG-mediated fusion in tumor tissue, numerous tumors derived from Clone $1 \mathrm{D}$ and $\mathrm{F}_{40}$ cell lines have been removed from their hosts, disrupted and filtered to produce single cell suspensions, and returned to culture for further analysis (Roos, 1985). In culture, these cells initially retained their high ether-lipid and low acyl-chain saturation, and all tumor explants (regardless of the cell line originally injected to generate the tumor) fused readily following PEG treatment -- as expected from the Clone 1D-like fatty acid saturation, but contrary to the $\mathrm{F}_{40}$-like ether lipid content. As the tumor explants were maintained in culture, each individual culture reverted to the initial lipid composition and fusion phenotype of the cell line originally injected: Clone 1D-derived tumor explants lost their ether-lipid with little effect on fusion response, while $\mathrm{F}_{40}$-derived tumor cells returned to their characteristic high degree of acyl chain saturation and lost their sensitivity to PEG. The combination of these results clearly links fusion response with acyl chain saturation rather than ether-lipid content.

\section{IMPLICATIONS OF THESE STUDIES}

\section{Applications of cell and membrane fusion}

The experiments described above demonstrate a functional association between lipid composition and cell membrane fusion. Through the use of defined media supplements it has been possible to completely control the response of these cells to a variety of fusogenic treatments. Although for any given cell line the extent of fusion induced by chemical agents (such as PEG)

\footnotetext{
*The muse of mice, a little-known illegitimate daughter of Mnemosyne (goddess of memory and mother of the Muses by Zeus).
} 
and enveloped viruses (such as MHV) are inversely correlated, appropriate lipid supplement experiments were able to control both phenomena at once. Thus, for example, parallel cultures of an intermediate fusion-altered cell line such as $\mathrm{F}_{16}$ can be made to mimic the PEG-sensitive/virus-resistant phenotype of Clone $1 \mathrm{D}$ by growth in high concentrations of polyunsaturated fatty acid, or to resemble the virus-sensitive/PEG-resistant character of $F_{40}$ cells, solely by growth in saturated fatty acid.

The ability to control the extent of membrane fusion virtually at will has numerous potential applications in the design of experimental protocols involving cell hybridization or other forms of membrane fusion (Roos et al., 1987). Even where the direct manipulation of fusion through lipid alteration may be undesirable or infeasible, the relationships between different types of fusion which have been elucidated above may provide useful clues to the selection of an appropriate fusogen. Careful studies on the extent of fusion between several of these cell lines which differ in their inherent responsiveness to PEG supplement existing models of cell fusion (Röhme and Thorburn, 1981), and provide a rational basis for maximizing desirable interactions in cell hybridization procedures (D.S. Roos, R.L. Davidson, and P.W. Choppin, manuscript in preparation).

\section{Membrane fusion mechanisms}

Several aspects of cellular lipid composition are associated with these fusion-altered cell lines, but the degree of acyl chain saturation and etherlipid content are most closely correlated with the observed differences in fusion phenotype. As previously noted (Roos and Choppin, 1985a), these two phenomena may be metabolically linked, and it is therefore not surprising

Table IV. Lipid Composition and Fusion Response of Tumor Explants

\begin{tabular}{|c|c|c|c|c|}
\hline \multicolumn{2}{|c|}{ Cell/Tissue sample* } & $\begin{array}{l}\text { FA Saturation } \\
\text { (S/P ratio) }\end{array}$ & Ether Lipid & $\begin{array}{l}\text { PEG Fusion } \\
\text { Response (\%) }\end{array}$ \\
\hline \multirow{2}{*}{$\begin{array}{l}\text { Clone } \\
\text { 1D }\end{array}$} & Control cells (prior to injection) & $1.0 \pm 0.1^{\dagger}$ & - & $94 \pm 5^{\dagger}$ \\
\hline & Tumor tissue & 1.1 & +++ & ND \\
\hline \multirow{8}{*}{$F_{40}\{$} & Tumor explants cultured $<2$ days & ND & ++ & 74 \\
\hline & Explants after 5 days in culture & 1.1 & - & 81 \\
\hline & Explants after 14 days in culture & 1.2 & - & 91 \\
\hline & Control cells (prior to injection) & $4.0 \pm 1.2^{\dagger}$ & ++ & $21 \pm 8^{\dagger}$ \\
\hline & Tumor tissue & 1.1 & +++ & ND \\
\hline & Tumor explants cultured $<2$ days & ND & ++ & 70 \\
\hline & Explants after 5 days in culture & 1.9 & ++ & 23 \\
\hline & Explants after 14 days in culture & 3.6 & ++ & 19 \\
\hline
\end{tabular}

$* 10^{6}$ Clone $1 \mathrm{D}$ or $\mathrm{F}_{40}$ cells were injected intramuscularly into 6-week-old irradiated $\mathrm{C} 3 \mathrm{HeB} / \mathrm{FeJ}$ mice, and tumors were removed for culture six weeks post-injection. Each data point is the average of results from at least three independent tumors. ND, not done.

$\dagger \pm$ standard deviation. 
that they have proven to be quite refractory to independent manipulation. Table $V$ presents a qualitative summary of the relevant results from a variety of experiments which have produced alterations in fatty acyl chain saturation, ether-lipid content, or both. Supplementation of the culture medium with fatty acids varying in their degree of saturation and conformational stereochemistry has allowed substantial control over acyl chain composition with lesser, but significant, variation in ether-lipid content. In these experiments, PEG fusion response can be dramatically altered (cf. Table III and Figure 2), although it is somewhat surprising that both cis and trans poly-

Table V. Cell Fusion and Lipid Composition: Summary of Lipid Alteration Experiments*

\begin{tabular}{lcccccc} 
& Treatment & & Ether Lipid & FA saturation & & PEG Fusion \\
\cline { 5 - 6 } & none & & - & - & + \\
$\mathrm{F}_{40}$ & $n$ & & + & + & -
\end{tabular}

\begin{tabular}{|c|c|c|c|c|}
\hline & Treatment & Ether Lipid & FA saturation & PEG Fusion \\
\hline Clone 1D & $+19: 0$ & $+1-$ & + & - \\
\hline$F_{40}$ & $+18: 3$ & $-1+$ & - & + \\
\hline
\end{tabular}

\begin{tabular}{|c|c|c|c|c|}
\hline & Treatment & Ether Lipid & FA saturation & PEG Fusion \\
\hline Clone 1D & $+18: 2$ trans & - & $\overline{\text { (but }} \mathbf{S}+\mathrm{t} P \boldsymbol{P} \uparrow$ ) & + \\
\hline$F_{40}$ & " " & $-1+$ & (but fluidity low) & + \\
\hline
\end{tabular}

\begin{tabular}{|c|c|c|c|c|}
\hline & Treatment & Ether Lipid & FA saturation & PEG Fusion \\
\hline Clone 1D & +FAOH (18:2) & + & $-1+$ & $+1-$ \\
\hline $\mathrm{F}_{40}$ & $"$ & + & + & - \\
\hline
\end{tabular}

\begin{tabular}{|c|c|c|c|c|}
\hline & Treatment & Ether Lipid & FA saturation & PEG Fusion \\
\hline Clone 1D & $40^{\circ} \mathrm{C}$ & - & $+1-$ & + \\
\hline$F_{40}$ & $29^{\circ} \mathrm{C}$ & $-1+$ & - & $-1+$ \\
\hline
\end{tabular}

\begin{tabular}{|c|c|c|c|c|}
\hline & Treatment & Ether Lipid & FA saturation & PEG Fusion \\
\hline Clone 1D & tumor explants & $+\rightarrow$ & - & + \\
\hline$F_{40}$ & $"$ & + & $\rightarrow+$ & $+\rightarrow-$ \\
\hline
\end{tabular}

* See text for descriptions of experimental protocols. 
unsaturates serve to stimulate fusion (Roos and Choppin, 1985b). Fatty alcohols, important precursors in ether-lipid biosynthesis (Hajra, 1983; Brown and Snyder, 1983; Friedberg et al., 1983), have also been used as a media supplement to increase ether-lipid levels (according to the procedures of Cabot and Snyder, 1980). Although ether-lipid production could be substantially stimulated in Clone 1D cells, no effect was observed on PEG-induced fusion beyond the level expected from accompanying changes in fatty acid saturation. Another experimental approach to the alteration of acyl chain saturation involves growing cells at different temperatures (Ferguson et al., 1975). The most useful experiments, however, emerged from the analysis of lipid composition and fusion response in cells derived from mouse tumors, as discussed above and in Table IV. Overall, PEG fusion response is most directly correlated with fatty acid saturation. Virus-induced fusion has only been examined in a limited number of these lipid alteration experiments, but wherever tested, virus fusion has been found to be inversely correlated with susceptibility to the fusogenic effects of PEG.

It is tempting, based on theoretical considerations and work on artificial systems, to base models for cell fusion on altered membrane fluidity (Ahkong et al., 1973; Kosower et al., 1975; Papahadjopoulos et al., 1979; Chaudhury and Ohki, 1981; Cullis et al., 1983). The central role of acyl-chain saturation in controlling fusion response superficially suggests that increased membrane fluidity increases susceptibility to PEG-induced fusion, while stiffer membranes predispose cells to virus-induced fusion. This interpretation is dfficult to reconcile, however, with the observation that both cis and trans polyunsaturates serve to promote PEG-induced fusion (Roos and Choppin, 1985b), despite the fact that trans polyunsaturated acyl chains stiffen the surface membranes of these cells as effectively as do saturated lipid supplements (unpublished data). It seems more likely that the role played by fatty acyl chains in controlling fusion is an indirect one, perhaps exerting an effect through interactions with membrane proteins. Such crucial controlling roles for lipids in regulating protein function have recently come to light in a number of systems (Sandermann, 1978; Cockroft and Gomperts, 1979; Benveniste and Vargaftig, 1983; Nishizuka, 1984; Low et al., 1986), and may also apply to cellular and viral proteins which induce membrane fusion.

Regardless of the precise biochemical basis (or bases) of cell fusion, the system described above provides a useful approach to the elucidation of whatever factors may be involved. It may be possible to use specific lipid species, known from these studies to relate to the control of cell fusion, as affinity reagents to isolate further fusion factors. Alternatively, taking advantage of the genetic basis of fusion alterations in these somatic cell mutants, it may be possible to directly search for the DNA sequences responsible for the fusionaltered phenotype of $\mathrm{F}_{40}$ cells.

\section{Use of membrane fusion mutants to study other membrane-associated phenomena}

The cell lines described in this report have proved to be useful in a number of studies unrelated to cell fusion (unpublished observations, and Roos and Choppin, 1984). Although the initial intent of these experiments was to select PEG fusion-resistant mutants, the result was to isolate cell lines which are progressively altered in several aspects of their membrane biochemistry. This panel of closely related cell lines can now be used to study other membrane-associated phenomena in addition to PEG-induced fusion. A partial listing of membrane interactions which preliminary studies have shown to vary across the series of increasingly PEG-resistant cell lines includes: fusion induced by various enveloped viruses, chemicals other than PEG, and 
spontaneously occurring fusion; pinocytosis and membrane recycling; viral infectivity, maturation, and transformation; bacterial and mycoplasmal invasion and pathogenesis; tumorigenicity, metastatic spread in vivo and in model systems; and immune recognition both in vivo and in vitro. As the cell lines described above are stable, homogeneous, and now partially characterized genetically and biochemically, it may be possible to extend the analysis of these phenomena to link particular aspects of their structure with these various functions, similar to the lipid-alteration studies which have so clearly linked acyl-chain saturation with fusion response.

ACKNOWLEDGEMENTS. I would like to express my thanks to Dr.s Purnell W. Choppin (The Rockefeller University, New York City, New York, and Howard Hughes Medical Institute, Bethesda, Maryland), Richard L. Davidson (Center for Genetics, University of Illinois Medical Center, Chicago, Illinois), and Kathryn V. Holmes (Department of Pathology, Uniformed Services University of the Health Sciences, Bethesda, Maryland) for their collaboration, advice, and support during the course of the studies described, and to Karen Winkel and Linda Wilkinson for able and enthusiastic technical assistance.

\section{REFERENCES}

Ahkong, Q.F., D. Fisher, W. Tampion, and J.A. Lucy, 1973, The fusion of erythrocytes by fatty acids, esters, retinol, and $\alpha$-tocopherol, Biochem. J. 136:147-155.

Benveniste, J., and B.B. Vargaftig, 1983, Platelet-activating factor: an ether lipid with biological activity, In: "Ether Lipids: Biochemical and Biomedical Aspects," H. Mangold and F. Paltauf, editors, pp. 355-376, Academic Press, New York.

Blow, A.M.J., A.J. Botham, D. Fisher, A.H. Goodall, C.P.S. Tilcock, and J.A. Lucy, 1978, Water and calcium ions in cell fusion induced by poly(ethylene glycol), FEBS Lett. 94:305-310.

Bollinger, J.N., 1967, The isolation and tentative identification of diacylglyceryl ethers from the Walker 256 carcinoma of the rat and a human lymphosarcoma, Lipids 2:143148.

Bratt, M.A., and W.R. Gallaher, 1969, Preliminary analysis of the requirements for fusion from within and fusion from without by Newcastle disease virus, Proc. Nat'l Acad. Sci. USA 64:536-543.

Brown, A.J., and F. Snyder, 1983, The method of alkyldihydroxyacetone-P synthase, J. Biol. Chem. 258:4184-4189.

Cabot, M.C., and F. Snyder, 1980, Manipulation of alkyklglycerolipid levels in cultured cells: fatty alcohol versus alkylglycerol supplements, Biochim. Biophys. Acta 617:410418.

Chaudhury, M.K. and S. Ohki, 1981, Correlation between membrane expansion and temperature-induced membrane fusion, Biochim. Biophys. Acta 642:365-374.

Choppin, P.W., and A. Scheid, 1980, The role of viral glycoproteins in adsorption, penetration, and pathogenicity of viruses, Rev. Infect. Dis. 2:40-61.

Cockroft, S., and B.D. Gomperts, 1979, Evidence for a role of phosphatidylinositol turnover in stimulus-secretion coupling, Biochem. J. 178:681-687.

Compans, R.W., K.V. Holmes, S. Dales, and P.W. Choppin, 1966, An electron microscopic study of moderate and virulent virus-cell interactions of the parainfluenza virus SV5, Virology 30:411-426.

Cullis, P.R., B. de Kruijff, M.J. Hope, A.J. Verkleij, R. Nayar, S.B. Farren, C. Tilcock, T.D. Madden, and M.B. Bally, 1983, Structural properties of lipids and their functional roles in biological membranes, In: "Membrane Fluidity in Biology," R.C. Aloia, editor, pp. 40-83, Academic Press, New York.

Davidson, R.L., 1977, Genetics of cultured mammalian cells, as studied by somatic cell hybridization, Nat'l Cancer Inst. Monograph 48:21-30.

Davidson, R.L., and P.S. Gerald, 1976, Improved techniques for the induction of mammalian somatic cell hybridization by polyethylene glycol, Somatic Cell Genet. 2:165-176.

Davidson, R.L., K.A. O'Malley, and T.B. Wheeler, 1976, Polyethylene glycol-induced mammalian cell hybridization: effect of polyethylene glycol molecular weight and concentration, Somatic Cell Genet. 2:271-280. 
de St. Groth, S.F., and D. Scheidegger, 1980, Production of monoclonal antibodies: strategy and tactics, J. Immunol. Methods 35:1-21.

Ferguson, KA., M. Glaser, W.H. Bayer, and P.R. Vagelos, 1975, Alteration of fatty acid composition of LM cells by lipid supplementation and temperature, Biochem. 14:146-151.

Friedberg, S.J., and M. Halpert, 1978, Ehrlich ascites tumor cell membranes: an abnormality in ether lipid content, J. Lipid Res. 19:57-64

Friedberg, S.J., S.T. Weintraub, M.R. Singer, and R.C. Greene, 1983, The mechanism of ether bond formation in O-alkyl lipid synthesis in Ehrlich ascites tumor, J. Biol. Chem. 258:136-142.

Furusawa, M., 1980, Cellular microinjection by cell fusion: technique and applications in biology and medicine, Int. Rev. Cytol. 62:29-67.

Gregoriades, G., editor, 1984, "Liposome Technology," CRC Press, Boca Raton, Florida.

Hajra, A.K, 1983, Biosynthesis of O-alkylglycerol ether lipids, In: "Ether Lipids: Biochemical and Biomedical Aspects," H. Mangold and F. Paltauf, editors, pp. 85-106, Academic Press, New York.

Helenius, A., J. Kartenbeck, K. Simons, and E. Fries, 1980, On the entry of Semliki Forest virus into BHK-21 cells, J. Cell Biol. 84:404-420.

Holmes, K.V., and P.W. Choppin, 1966, On the role of the response of the cell membrane in determining virus virulence. Contrasting effects of the parainfluenza virus SV5 in two cell types, J. Exp. Med. 124:501-520.

Holmes, K.V., E.W. Doller, and L.A. Sturman, 1981, Tunicamycin resistant glycolsylation of a coronavirus glycoprotein: demonstration of a novel type of viral glycoprotein, Virology 115:334-344.

Kit, S., D.R. Dubbs, L.J. Piekarski, and T.C. Hsu, 1963, Deletion of thymidine kinase activity from L cells resistant to bromodeoxyuridine, Exp. Cell Res. 31:297-312.

Kosower, N.S., E.M. Kosower, and P. Wegman, 1975, Membrane mobility agents II. Active promoters of cell fusion, Biochim. Biophys. Acta 401:530-534.

Langerhans, T., 1868, Über Riesenzellen mit wandständigen Kernen in Tuberkeln und die fibröse Form des Tuberkels, Virchow's Arch. Path. Anat. Physiol. 42:382-404.

Lin, H.J., F.C.S. Ho, and C.L.H. Lee, 1978, Abnormal distribution of O-alkyl groups in the neutral glycerolipids from human hepatocellular carcinomas, Cancer Res. 38:946-949.

Low, M.G., M.A.J. Ferguson, A.H. Futerman, and I. Silman, 1986, Covalently attached phophatidylinositol as a hydrophobic anchor for membrane proteins, Trends Biochem. Sci. 11:212-215.

Lucy, J.A., 1982, Biomembrane fusion, In: "Biological Membranes," Vol. 4 (D. Chapman, editor, pp. 367-415, Academic Press, London.

Mangold, H, and F. Paltauf, editors, 1983, "Ether Lipids: Biochemical and Biomedical Aspects," Academic Press, New York.

Nishizuka, Y., 1984, The role of protein kinase $C$ in cell surface signal transduction and tumor promotion, Nature (Lond.) 308:693-698.

O'Malley, K.A, and R.L. Davidson, 1977, A new dimension in suspension fusion techniques with polyethylene glycol, Somatic Cell Genet. 3:441-448.

Papahadjopoulos, D., G. Poste, and W.J. Vail, 1979, Studies on membrane fusion with natural and model membranes, Methods Membr. Biol. 10:1-121.

Pfleger, R.C., N.G. Anderson, and F. Snyder, 1968, Lipid class composition and fatty acid composition of rat liver plasma membranes isolated by zonal centrifugation, Biochemistry 7:2826-2833.

Poste, G., and G.L. Nicholson, editors, 1978, "Membrane Fusion," Cell Surface Reviews, Vol. 5, Elsevier/North Holland, Amsterdam.

Robinson, J.M., D.S. Roos, R.L. Davidson, and M.L. Karnovsky, 1979, Membrane alterations and other morphological features associated with polyethylene glycol-induced cell fusion, J. Cell Sci. 40:63-75.

Röhme, D., and D. Thorburn, 1981, Quantitative cell fusion: derivation and application of theoretical models, Somatic Cell Genet. 7:43-57.

Roos, D.S., 1985, "Membrane Fusion, Lipid Composition, and Tumorigenicity of Cultured Cells," Ph.D. thesis, pp. 201+xviii, The Rockefeller University, New York.

Roos, D.S., and P.W. Choppin, 1984, Tumorigenicity of cell lines with altered lipid composition, Proc. Nat'l Acad. Sci. USA 81:7622-7626.

Roos, D.S., and P.W. Choppin, 1985a, Biochemical studies on cell fusion I. Lipid composition of fusion-resistant cells, J. Cell Biol. 101:1578-1590.

Roos, D.S., and P.W. Choppin, 1985b, Biochemical studies on cell fusion II. Control of fusion response by lipid alteration, J. Cell Biol. 101:1591-1598. 
Roos, D.S., and R.L. Davidson, 1980, Isolation of mouse cell lines resistant to the fusioninducing effect of polyethylene glycol, Somatic Cell Genet. 6:381-390.

Roos, D.S., J.M. Robinson, and R.L. Davidson, 1983, Cell fusion and intramembrane particle distribution in polyethylene glycol-resistant cells, J. Cell Biol. 87:909-917.

Roos, D.S., R.L. Davidson, and P.W. Choppin, 1987, Control of cell fusion in polyethylene glycol-resistant cell mutants: applications to fusion technology, In: "Cell Fusion," A.E. Sowers, editor, pp. 123-144, Plenum Press, New York.

Sandermann, H., 1978, Regulation of membrane enzymes by lipids, Biochim. Biophys. Acta 515:209-237.

Schroeder, F., and P.R. Vagelos, 1976, Effects of phospholipid base analogs on the subcellular membrane ether composition of suspension cultured LM cells, Biochim. Biophys. Acta 441:239-254.

Schlegel, R.A., and M.R. Lieber, 1987, Microinjection of culture cells via fusion with loaded erythrocytes, In: "Cell Fusion," A.E. Sowers, editor, pp. 457-478, Plenum Press, New York.

Snyder, F., editor, 1972, "Ether Lipids: Chemistry and Biology," Academic Press, New York.

Sowers, A.E., editor, 1987, Cell Fusion, Plenum Press, New York.

Spear, P.G., 1987, Virus-induced cell fusion, In: "Cell Fusion," A.E. Sowers, editor, pp. 3-32, Plenum Press, New York.

Spector, A.A., S.N. Mathur, T.L. Kaduce, and B.T. Hyman, 1981, Lipid nutrition and metabolism of cultured mammalian cells, Prog. Lipid Res. 19:155-186.

Wang, E.W., D.S. Roos, M.H. Heggeness, and P.W. Choppin, 1982, Function of cytoplasmic fibers in syncytia, Cold Spring Harbor Symp. Quant. Biol. 46:997-1012.

White, J., M. Matlin, and A. Helenius, 1981, Cell fusion by Semliki Forest, influenza, and vesicular stomatitis virus, J. Cell Biol. 89:674-679.

White, J., M. Kielian, and A. Helenius, 1983, Membrane fusion proteins of enveloped animal viruses, Quart. Rev. Biophys. 16:151-195.

Wood, R., and F. Snyder, 1967, Characterization and identification of glyceryl ether diesters present in tumor cells, J. Lipid Res. 8:494-500.

Wood, R., N.G. Anderson, and D.C. Swartzendruber, 1970, Characterization of lipids isolated from membranous material, Arch. Biochem. Biophys. 14:190-197.

Zimmermann, U., 1982, Electric field-mediated fusion and related electrical phenomena, Biochim. Biophys. Acta 694:227-277. 